\title{
Síndrome de Noonan asociado a subclavia aberrante y secuestro pulmonar
}

\author{
Noonan syndrome associated with aberrant right \\ subclavia and pulmonary sequestration
}

\author{
Marvin Vladimir Solís-Trujeque,* Israel Didier Cruz-Anleu*
}

*Centro Regional de Alta Especialidad, Hospital de Especialidades Pediátricas, Tuxtla Gutiérrez, Chiapas, México.

\begin{abstract}
RESUMEN. El síndrome de Noonan es una enfermedad de herencia autosómica dominante. Las malformaciones respiratorias más comunes son el pectum carinatum, el pectum excavatum, la linfangiectasia pulmonar y el quilotórax. También se ha relacionado con malformaciones cardiovasculares, como la estenosis valvular pulmonar, la coartación aórtica y la estenosis de ramas pulmonares. Se presenta el caso de un masculino de seis años, con diagnóstico de arteria subclavia derecha aberrante, secuestro pulmonar y síndrome de Noonan, que es enviado para su valoración con diagnóstico de asma. Se realizó una revisión de la literatura en diversas revistas médicas en búsqueda de alguna asociación o caso previo. Concluimos que el diagnóstico es complejo, puesto que no existe en la literatura una relación entre estas patologías. Por lo que es importante realizar una historia clínica y exploración física oportunas, así como estudios de imagen adecuados en niños con este síndrome.
\end{abstract}

Palabras clave: Síndrome de Noonan, secuestro pulmonar, arteria subclavia derecha aberrante, malformación broncopulmonar.

\section{INTRODUCCIÓN}

El síndrome de Noonan ( $\mathrm{SN}$ ) es una enfermedad de herencia autosómica dominante, se estima una incidencia de 1/1,000 a 1/2,500 recién nacidos. Las malformaciones respiratorias más comunes son el pectum carinatum superior y/o el excavatum inferior, puede haber linfangiectasia pulmonar o quilotórax. ${ }^{1,2}$ Se relaciona en $50-80 \%$ a car-

Correspondencia:

Dr. Israel Didier Cruz-Anleu

Centro Regional de Alta Especialidad.

Hospital de Especialidades Pediátricas,

Tuxtla Gutiérrez, Chiapas, México.

Correo electrónico: canleu2@yahoo.com.mx

Recibido: 24-IX-2020; aceptado: 18-XI-2020.

Citar como: Solís-Trujeque MV, Cruz-Anleu ID. Síndrome de Noonan asociado a subclavia aberrante y secuestro pulmonar. Neumol Cir Torax. 2021; 80 (1): 51-55. https://dx.doi.org/10.35366/99455
ABSTRACT. Noonan syndrome is an autosomal dominant inherited disease. The most common respiratory malformations are pectum carinatum, pectum excavatum, pulmonary lymphangiectasia, and chylothorax. It has also been associated with cardiovascular malformations, such as pulmonary valve stenosis, aortic coarctation, and pulmonary branch stenosis. We present the case of a 6-year-old male, with a diagnosis of aberrant right subclavian artery, pulmonary sequestration and Noonan syndrome, sent for evaluation with a diagnosis of asthma. A review of the literature was carried out in various medical journals in search of some association or previous case. We conclude that the diagnosis is complex, since there is no association in the literature between these pathologies. Therefore, it is important to carry out an adequate medical history and physical examination, as well as adequate imaging studies in children with this syndrome.

Keywords: Noonan syndrome, pulmonary sequestration, aberrant right subclavian artery, bronchopulmonary malformation.

diopatías congénitas, la más predominante es la estenosis valvular pulmonar, seguida de la coartación aórtica y de la estenosis de ramas pulmonares. ${ }^{1}$

El objetivo de este artículo es presentar el caso de un escolar con diagnóstico de SN y arteria subclavia derecha aberrante más secuestro pulmonar intralobar derecho. Se realizó una revisión de la literatura.

\section{PRESENTACIÓN DEL CASO}

Se trata de masculino de seis años de edad con diagnóstico de SN, el cual es visto por primera vez en la consulta de neumología a esta edad, con diagnóstico de envío de asma de difícil control y neumonía. Los antecedentes mencionan ser producto de la segunda gesta, madre con eclampsia, obtenido por vía cesárea, prematuro de 36 semanas de gestación, con peso de 2,600 g y Apgar 8. Esquema de vacunación incompleto. Fue valorado por cardiología, quien refiere un electrocardiograma (EKG): ritmo sinusal, sin trastornos de conducción ni repolarización. 


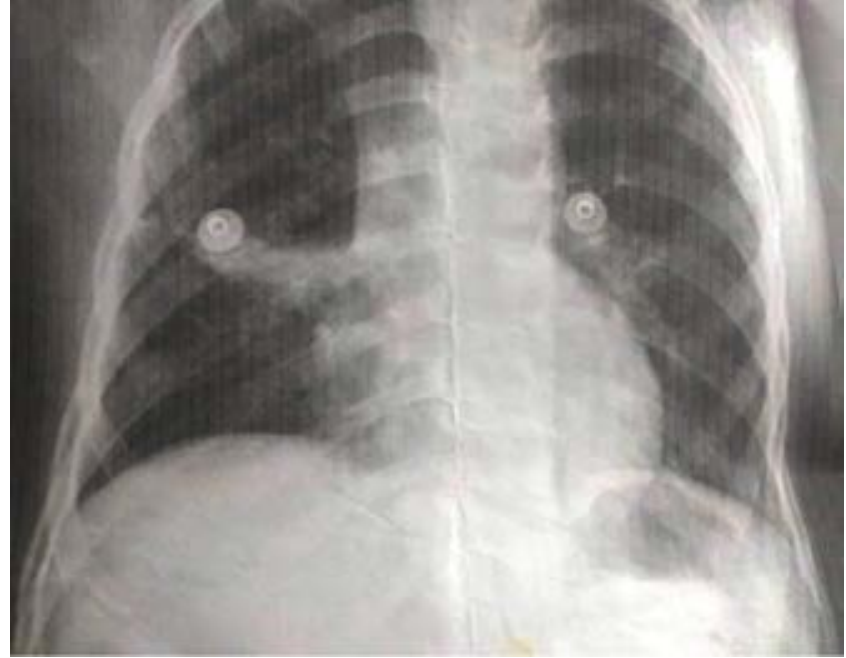

Figura 1: Telerradiografía de tórax: presenta una imagen que incrementa la densidad pulmonar en el lóbulo medio caracterizada por un patrón de consolidación con broncograma aéreo y signo de silueta positivo, sugerentes de una atelectasia pulmonar.

Ecocardiografía: presión sistólica arterial pulmonar (PSAP): $25 \mathrm{mmHg}$ y fracción de eyección del ventrículo izquierdo (FEVI) 61\%, arteria pulmonar y drenaje venoso pulmonar normal. Los síntomas predominantes son tos crónica, sibilancias, disnea de medianos esfuerzos y disfagia a sólidos de cinco años cuatro meses de evolución. Exploración física: índice de masa corporal: 15.2, $\mathrm{SpO}_{2}$ : 96\%. Morfología del tórax normal, diámetro intermamilar $16 \mathrm{~cm}$, no se integra síndrome pleuropulmonar alguno. Tratado con esteroide inhalado y broncodilatador de larga acción, con mejoría de la sintomatología.

Estudios: difusión de monóxido de carbono (DLCO) $7.5 \mathrm{~mL} / \mathrm{min} / \mathrm{mmHg}$ (valor predicho 10.2), espirometría posbroncodilatador con una respuesta en volumen espiratorio forzado en el primer segundo $\left(\mathrm{FEV}_{1}\right)$ del $16 \%$ y de más de $200 \mathrm{~mL}$. La radiografía de tórax muestra un patrón atelectásico (Figura 1). Serie esofagogastroduodenal (SEGD) con presencia de reflujo grado III; en la angiotomografía de tórax (angio-TC) se observa una subclavia derecha aberrante (Figura 2), lo mismo que en la aorta descendente, también un vaso aberrante (Figuras 3 y 4).

\section{DISCUSIÓN}

El término anillo vascular se utiliza para describir la combinación de estructuras vasculares, y en ocasiones ligamentosas, del arco aórtico y sus ramas que encierran a la tráquea y al esófago, lo que causa un efecto de masa y obstrucción de las vías respiratorias o del esófago y genera dificultad en la respiración o en la deglución. ${ }^{3}$ Son relativamente raros con una incidencia de 2-10/10,000 habitantes y son menos de $1 \%$ de las anomalías congénitas cardiovasculares. ${ }^{4}$ Lo ideal es realizar el diagnóstico a edades más tempranas de la vida, puesto que pueden producir alteraciones en la calidad de vida del paciente y sus familiares, lo cual altera la dinámica familiar con base en el síntoma predominante. Tal y como sucedió en este caso, la manifestación de dificultad respiratoria y sibilancias Ilevaron al paciente a sufrir diversas hospitalizaciones y al final a complicarse con neumonía y atelectasias, síntomas y signos que obligaron su traslado a especialidad respiratoria infantil.

Durante el crecimiento embrionario, en la cuarta semana, aparecen hendiduras faríngeas irrigadas por seis pares de arcos aórticos primitivos, el sistema de arcos aórticos pierde gradualmente su disposición simétrica original, se desarrollan y retroceden con el desarrollo de las bolsas faríngeas. La persistencia o involución de estos arcos determinan las diferentes variedades de anillos vasculares. ${ }^{4}$ Existe una clasificación de los anillos vasculares propuesta
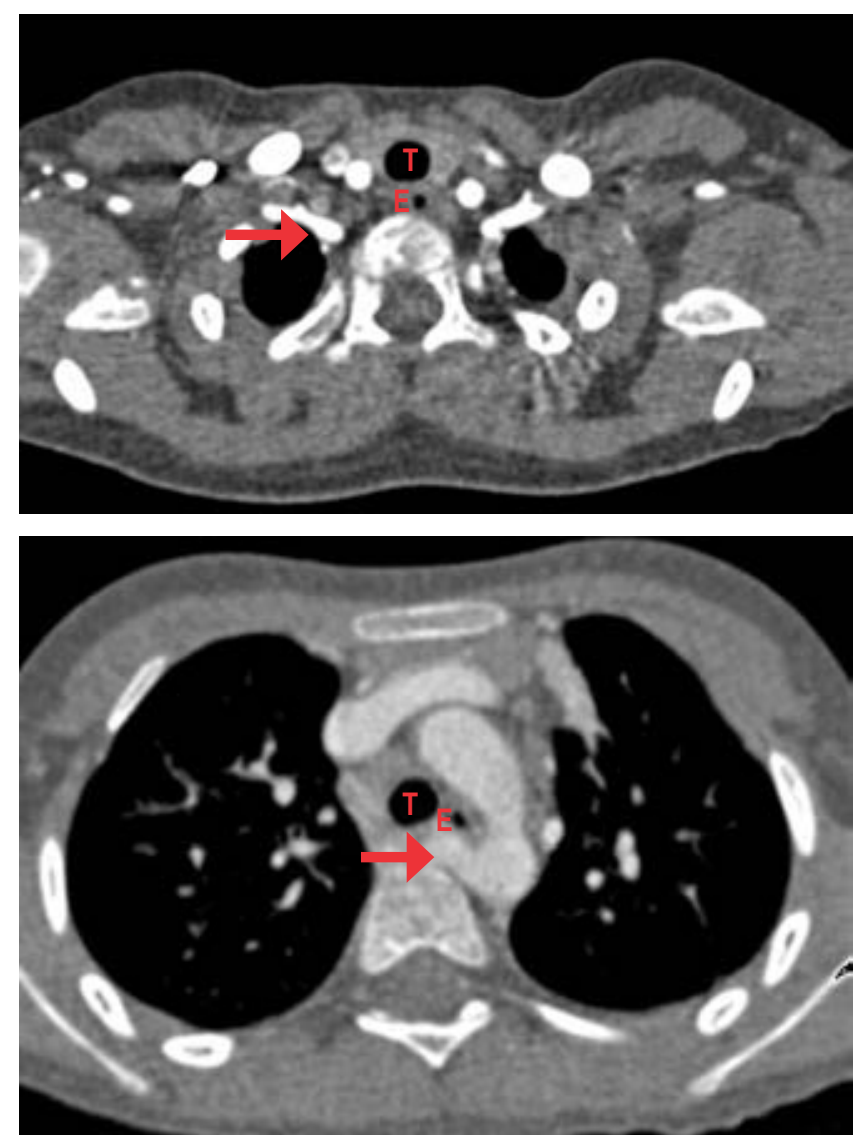

Figura 2: Angiotomografía computarizada de tórax fase arterial: se observa la presencia de subclavia derecha aberrante (flecha) que discurre desde la cara posterior de porción proximal de aorta descendente hacia la derecha del paciente por detrás del esófago (E) y que reduce el calibre del mismo en forma considerable. 

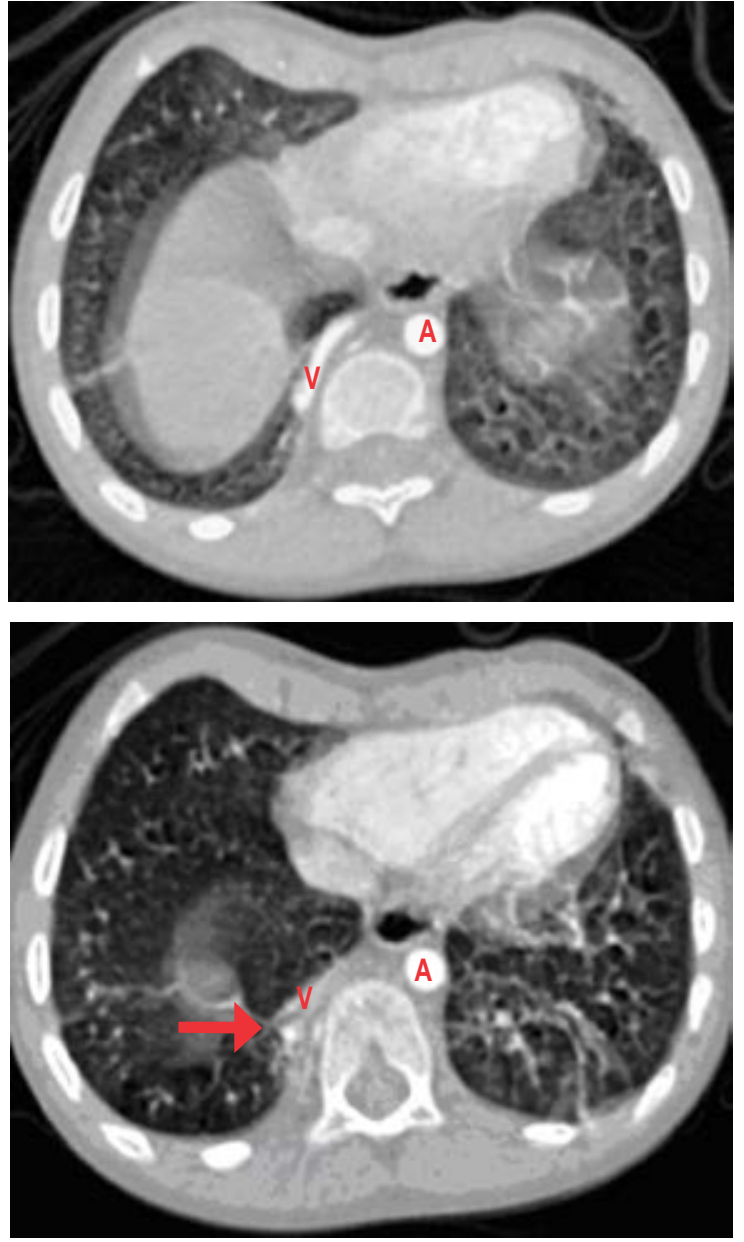

Figura 3: Angiotomografía computarizada de tórax en la cual se observa la presencia de hipervascularidad pulmonar que genera una lesión sólida muy pequeña (flecha) y vaso nutricio $(\mathrm{V})$ que nace de la aorta torácica descendente $(\mathrm{A})$ y se localiza cerca del diafragma, de $4 \mathrm{~mm}$ de diámetro que se dirige a la región basal del pulmón derecho.

por Stewart et al. en la cual las anomalías vasculares pueden consistir en anillos completos (doble arco aórtico) que pueden comprimir la tráquea y el esófago, o bien incompletos (arteria subclavia aberrante derecha) que ocasionalmente comprime el esófago. ${ }^{5}$ En nuestro paciente la disfagia fue un síntoma que tuvo su aparición (junto con las sibilancias) en una etapa muy temprana de la vida, la cual se asoció con la ablactación del paciente. Este dato corrobora lo observado en la literatura, donde este tipo de malformación de la subclavia derecha tiende a producir la compresión esofágica y manifestarse en diversos grados de disfagia y hasta regurgitaciones.

La arteria subclavia derecha aberrante ha sido reconocida y encontrada en $0.4 \%$ de las autopsias y se ha asociado con trisomía 21 y otros síndromes genéticos asociados con anomalías del arco aórtico, ${ }^{6}$ lo que la hace uno de los anillos vasculares menos comunes. Sus signos y síntomas inician en los primeros meses de vida y son característicos: estridor bifásico o espiratorio que aumenta con el llanto y la alimentación, «tos seca», infecciones respiratorias recurrentes, episodios de apnea refleja y cianosis, sibilancias, hiperextensión cervical y retracción esternal e intercostal. Por lo común, dichos síntomas son intermitentes, se modifican según posición y pueden incluso confundirse con un asma de difícil control, ${ }^{2}$ tal y como sucedió en el caso previamente descrito. Cuando resulta comprometida la vía digestiva por compresión del esófago, los síntomas más frecuentes son disfagia, vómitos, desnutrición y dificultad en
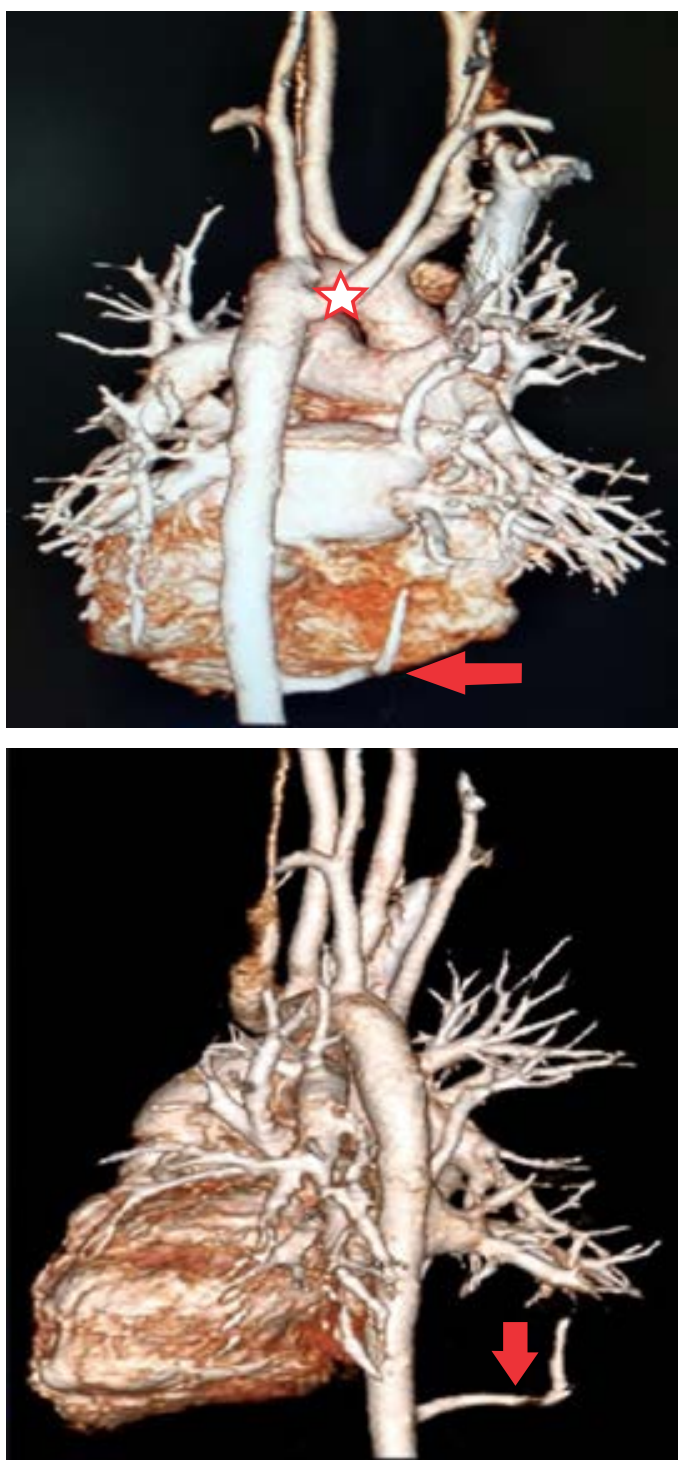

Figura 4: Reconstrucción vascular en la cual se observa la presencia de la arteria nutricia de secuestro pulmonar intralobar (flecha), la cual se origina de la aorta descendente torácica y vaso aberrante subclavia derecha (estrella). 
la alimentación, principalmente con la ingesta de sólidos. ${ }^{8}$ En cuanto a la sintomatología descrita, la disfagia, que es uno de los síntomas cardinales para el diagnóstico, fue subestimada por los padres hasta que se realizó la evaluación.

Por otra parte, las técnicas de imagen son pruebas complementarias que confirmarán el diagnóstico. La radiografía de tórax puede proporcionar información sobre el desplazamiento del arco aórtico o la tráquea, la presencia de una estenosis traqueal, así como infiltraciones pulmonares, distelectasias o atelectasias como signos de compresión de las vías respiratorias. ${ }^{9}$ El esofagograma muestra la indentación esofágica; sin embargo, la información anatómica obtenida no es lo suficientemente precisa para el tratamiento terapéutico, por lo que se requieren imágenes transversales como la tomografía computarizada (TC) o la resonancia magnética (RM). Se pueden usar métodos de posprocesamiento para generar datos $3 \mathrm{D}$, tanto de la TC como de la RM, dando una vista detallada de la morfología vascular..$^{10}$ En cuanto a los estudios de radiología la SECD no reveló identaciones esofágicas, sólo la presencia del reflujo severo; tampoco la tele de tórax mostró imágenes radiológicas sugerentes de las malformaciones, por lo que no fueron concluyentes para el diagnóstico. Por lo tanto, la TC de tórax y la angiotomografía son los estudios de elección para el diagnóstico de los anillos vasculares y de las malformaciones pulmonares como lo es el secuestro pulmonar intralobar o extralobar, la otra patología agregada en nuestro paciente.

En cuanto al secuestro pulmonar intralobar y su asociación con este síndrome no existe bibliografía a nivel mundial que los relacione en una entidad común. El secuestro pulmonar es una malformación congénita broncopulmonar poco frecuente caracterizado por un segmento anormal pulmonar irrigado por una arteria aberrante de origen sistémico, en 95\% de los casos se ubica en el lóbulo inferior izquierdo, representan de $0.15-6.4 \%$ de todas las malformaciones pulmonares congénitas. ${ }^{11,12}$ La incidencia anual estimada es de 30 a 42 casos por 100,000 habitantes..$^{13}$ Es frecuente que se asocien con otras anomalías congénitas como la hernia diafragmática o la cardiopatía congénita. ${ }^{14}$ Se clasifica en intralobar y extralobar de acuerdo con la ausencia o presencia de una cubierta de pleura visceral independiente en tejidos pulmonares anormales. ${ }^{12} \mathrm{El}$ secuestro intralobar es más común, se presenta en $75 \%$ de los casos. ${ }^{13}$ Estas estadísticas hacen de este caso algo poco común, puesto que a pesar de que el secuestro pulmonar intralobar es más común, la localización derecha puede hacer más difícil su diagnóstico.

Los síntomas de esta enfermedad son atípicos y tienden a diagnosticarse erróneamente, los pacientes presentan cuadros repetitivos de neumonías. Se ha descrito neumotórax de repetición, bronquitis obstructiva y dolor torácico, aunque menos frecuentes. ${ }^{13}$ En las radiografías de tórax, el secuestro intralobar puede contener aire, suelen ser más heterogéneos y menos definidos y pueden imitar una neumonía o un absceso pulmonar. En las tomografías se puede observar una masa quística con contenido aéreo o líquido, enfisema focal e hipervascularización del tejido afectado. ${ }^{13}$ La presencia del vaso anómalo se demuestra con tomografía con contraste intravenoso en $72 \%$ de la aorta torácica descendente, de la aorta abdominal, el eje celíaco o la arteria esplénica (21\%), arteria intercostal $(3 \%)$ y rara vez a través del subclavio, torácica interna y arterias pericardiacofrénicas. ${ }^{15}$ Tal y como se ha mencionado, los estudios radiológicos son sumamente importantes y aunque la radiografía no fue concluyente para el diagnóstico de este caso, la angio-TC sí lo fue acorde a lo que marca la literatura, en donde se observó un aumento en la hipervascularización, así como la presencia de un vaso anómalo de la aorta torácica descendente. El tratamiento de consenso para los secuestros intralobares y extralobares es la remoción quirúrgica. ${ }^{11-15}$ Este paciente se encuentra en espera para realizarle embolización por cateterismo cardíaco con un dispositivo oclusor.

\section{CONCLUSIONES}

El diagnóstico es complejo, sobre todo porque no existe en la literatura una asociación entre las patologías descritas. Es muy importante hacer una adecuada valoración y seguimiento al realizar estudios de imagen adecuados y una historia clínica completa, sin minimizar ningún síntoma digestivo y respiratorio. De acuerdo con la revisión bibliográfica no se encontró ninguna asociación entre el $\mathrm{SN}$ y los diagnósticos finales del paciente, lo que hace de éste un caso único.

\section{REFERENCIAS}

1. Ballesta-Martínez MJ, Guillén-Navarro E. Síndrome de Noonan. Protoc Diagn Ter Pediatr [Internet]. 2010 [Fecha de acceso: 18 de mayo de 2020];1:56-63. Disponible en: https://www.aeped.es/sites/ default/files/documentos/sindrome_de_noonan.pdf

2. Martínez-León MI, Ceres-Ruiz L, Solbes-Vila R, Valls-Moreno E. Síndrome de Noonan y quilotórax. Radiologia. 2001;43(4):197-201. doi: 10.1016/S0033-8338(01)76955-X.

3. Maggiolo MJ, Rubilar OL, Abara ES. Anillos vasculares en pediatría. Presentación de 2 casos. Neumol Pediatr. 2017;12(3):128-132.

4. Rioseco SP, Ahumada OA, Vásquez GD. Anillos vasculares como diagnóstico diferencial del asma. Rev Chil Enf Respir. 2012;28(1):5157. http://dx.doi.org/10.4067/S0717-73482012000100007

5. Hagler DJ, Bowman J, editors. Vascular rings and pulmonary slings in visual guide to neonatal cardiology. Nueva Jersey, Estados Unidos: John Wiley \& Sons Ltd; 2018 [Access date: 2020 May 26]. Available from: https://onlinelibrary.wiley.com/doi/pdf/10.1002/9781118635520. $\operatorname{ch} 43$

6. Zanetta A, Cuestas G, Rodríguez H, Tiscornia C. Anillos vasculares: obstrucción de vía aérea en niños. Serie de casos. Arch Argent 
11. Bracho MF, Pizarro TG, Sepúlveda CJ. Secuestro pulmonar en un lactante. Rev Chil Pediatr. 2007;78(5):494-499.

73482012000100007

7. Bordino L, Penida F, Scatolini ML, Cocciaglia A, Botto HA, Rodríguez HA. Anillos vasculares. Serie de 28 pacientes atendidos en un Servicio de Endoscopia Respiratoria Pediátrica. Arch Argent Pediatr. 2019;117(4):e393-e398. http://dx.doi.org/10.4067/S071773482012000100007

8. Mijangos-Vázquez R, Patiño-Bahena E, Martínez-García A, Herrera $\mathrm{J}$, Calderón-Colmenero J, Buendía-Hernández A, et al. Arteria subclavia derecha aberrante en niños examinados en el Instituto Nacional de Cardiología Ignacio Chávez (1992-2012). Arch Cardiol Méx. 2014;84(3):155-161. https://doi.org/10.1016/j.acmx.2013.10.010

9. Tola H, Ozturk E, Yildiz O, Erek E, Haydin S, Turkvatan A, et al. Assessment of children with vascular ring. Pediatr Int. 2017;59(2):134140. https://doi.org/10.1111/ped.13101

10. Kir M, Saylam GS, Karadas U, Yilmaz N, Cakmakci H, Uzuner N, et al. Vascular rings: Presentation, imaging strategies, treatment, and outcome. Pediatr Cardiol. 2012;33(4):607-617. https://doi.org/10.1007/ s00246-012-0187- $x$
12. Ou J, Lei X, Fu Z, Huang Y, Liu E, Luo Z, et al. Pulmonary sequestration in children: a clinical analysis of 48 cases. Int $\mathrm{J}$ Clin Exp Med. 2014;7(5):1355-1365.

13. De León-Ureña ZA, Sadowinski-Pine S, Jamaica-Balderas L, Penchyna-Grub J. Secuestro pulmonar asociado a una malformación congénita de la vía aérea pulmonar. Bol Med Hosp Infant Mex. 2018;75:119-126. https://doi.org/10.24875/BMHIM.M18000007

14. Sfakianaki AK, Copel JA. Congenital cystic lesions of the lung: congenital cystic adenomatoid malformation and bronchopulmonary sequestration. Rev Obstet Gynecol. 2012;5(2):85-93.

15. Wani SA, Mufti GN, Bhat NA, Baba AA. Pulmonary sequestration: early diagnosis and management. Case Rep Pediatr. 2015;2015:454860. doi: 10.1155/2015/454860. intereses.
Conflicto de intereses: Los autores declaran no tener conflicto de 